\title{
The Versatile FEG-SEM: From Ultra-High Resolution To Ultra-High Surface Sensitivity
}

Jingyue Liu

Science \&Technology, Monsanto Company, 800 N. Lindbergh Blvd., St. Louis, MO 63167, USA.

The use of a high-brightness field-emission gun (FEG), new lens designs, and novel detection systems in the SEM makes it possible to routinely examine nanometer or sub-nanometer surface features of bulk samples. Figure 1, for example, shows a high-resolution secondary electron (SE) image of a carbon-supported Pt sample, clearly revealing the size and spatial distribution of the $\mathrm{Pt}$ nanoparticles as well as the detailed pore structure of the carbon support. This type of detailed information is invaluable for characterizing challenging materials problems such as heterogeneous catalysts. Not only SE but also backscattered electron (BE) signals can be collected to form highresolution images. The energy of the primary electrons in a modern FEG-SEM can be varied from about $30 \mathrm{keV}$ to $100 \mathrm{eV}$, still providing high spatial resolution. The use of Cs- and Cc-correctors in the future generation FEG-SEMs can provide much smaller probe sizes with higher beam currents, further expanding the application of FEG-SEMs to challenging materials problems.

The use of low energy electrons is especially valuable and makes it possible to directly observe nonconducting materials with high resolution and reduced beam damage. Low voltage SEM is now the preferred mode of operation for examining non-conducting or delicate materials. Nanometer scale resolution is achievable with primary electron energies as low as $1 \mathrm{keV}$. Figure $2 \mathrm{a}$ shows a SE image of a gold film supported on graphite, clearly revealing the formation of faceted nano-holes in the gold film. Since electrons with energies in the range of 20-100 eV strongly interact with solid materials, we expect that highly surface-sensitive signals can be generated with the use of extremely low-energy electrons.

A recent development is the use of a retarding field to modulate the landing energy of the primary electrons [1]. By simply applying a negative potential to the specimen, a retarding field can be generated between the specimen and a grounded electrode above the specimen. With this arrangement the specimen itself is part of a "cathode lens" system and high-resolution images can be obtained with very low-energy electrons [2]. Figure $2 b$ shows such an image of the supported gold film, obtained with the landing energy of the electron probe of only $100 \mathrm{eV}$. Figure $3 \mathrm{a}$ and $3 \mathrm{~b}$ show SEM images of gold nanoparticles supported on a slightly oxidized silicon substrate, obtained with electron landing energies of $100 \mathrm{eV}$ and $50 \mathrm{eV}$, respectively. The unusual bright contrast of some gold nanoparticles may originate from the effect of low-energy electron diffraction or from the local charging of the gold nanoparticles. The signal strength decreases rapidly when the landing energy of the primary electrons falls well below $50 \mathrm{eV}$.

The ultimate resolution of ultra-low-voltage (ULV) SEM images is determined by the combined properties of the probe-forming lens and the cathode lens. Although the SE yield decreases rapidly with decreasing energy for electrons with energies $<100 \mathrm{eV}$, the total reflection of the primary electrons increases with decreasing electron energy. At very low eenergies, significant contrast variations may occur and the collected signal is highly sensitive to surface inhomogeneities and modifications such as variations in work function, surface steps, and local patch fields. Figure 4 shows images of a mica surface obtained with electron landing energies of $500 \mathrm{eV}, 12 \mathrm{eV}$, and $3 \mathrm{eV}$, respectively. The contrast reversal of the surface steps is clearly visible. The contrast of ULV images also strongly depends on the specific SE detection configuration and the electrical field 
distribution on and near the sample surface. The contrast mechanisms, the image resolution, and the applications of various high-resolution SEM imaging techniques will be discussed.

References

[1] L. Frank and I. Mullerova, J. Electron Microsc. 48 (1999) 205.

[2] J. Liu, Int. J. Mod. Phys. B16 (2002) 4387.

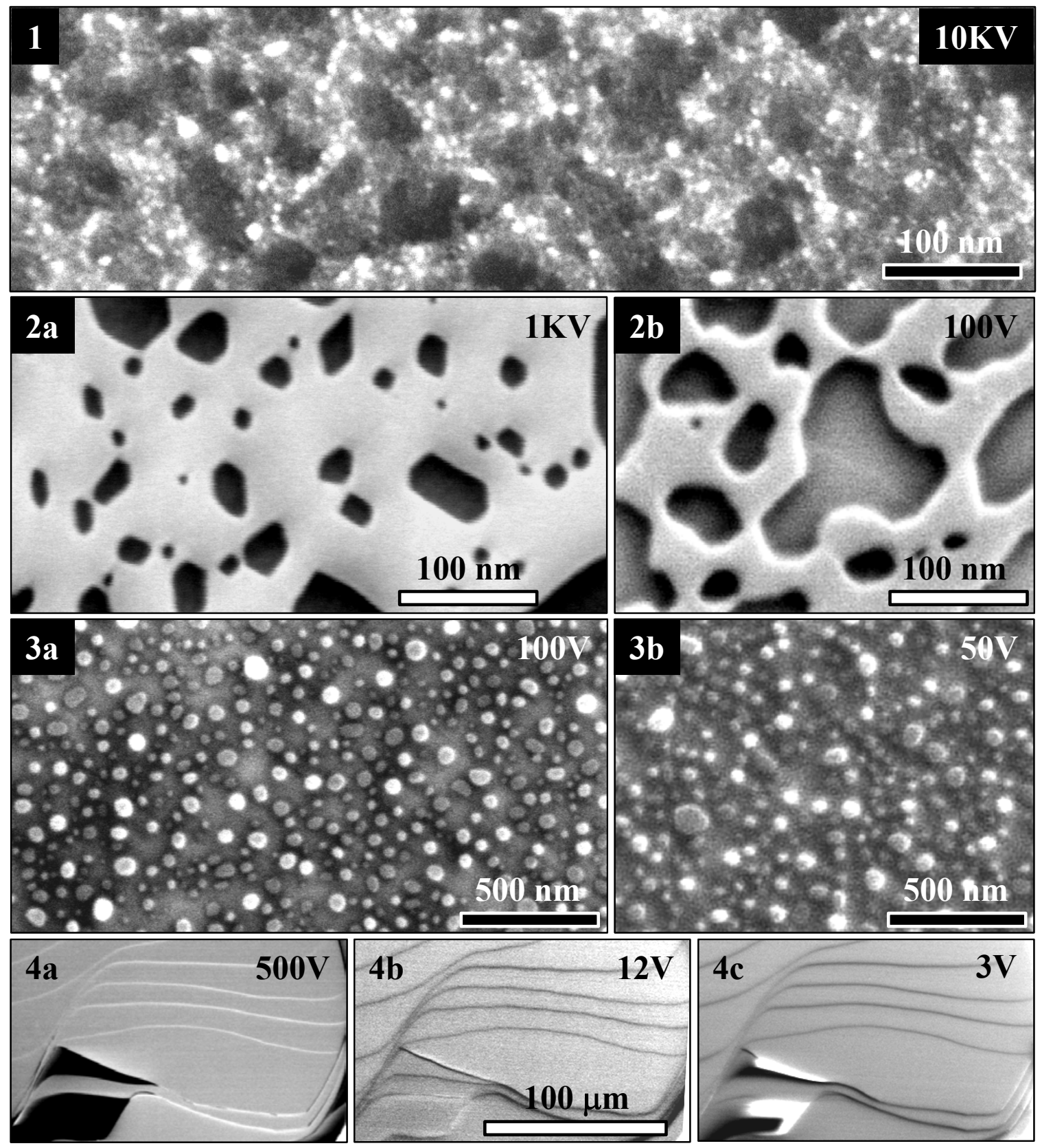

Fig. 1 High-resolution SE image of $\mathrm{Pt} / \mathrm{C}$ revealing $\mathrm{Pt}$ nanoparticles and structure of carbon pores. Fig. 2 LV (a) and ULV (b) SE images of gold film showing faceted holes.

Fig. 3 ULV SEM images of Au nanoparticles on oxidized silicon showing unusual particle contrast. Fig. 4 LV (a) and ULV SEM images of mica surface show reversal of step contrast. 